\title{
EL MARCO JURÍDICO DE LAS FUERZAS ARMADAS ESPAÑOLAS EN CONTEXTOS OPERATIVOS
}

\section{THE LEGAL FRAMEWORK OF THE SPANISH ARMED FORCES IN OPERATIONAL CONTEXTS}

\author{
Ángel Serrano BarberáN* \\ Investigador independiente, España \\ lithasaldana@hotmail.com
}

\section{RESUMEN:}

Las Fuerzas Armadas españolas, constituidas por el Ejército de Tierra, la Armada y el Ejército del Aire, tienen constitucionalmente asignadas una serie de misiones, en el desarrollo de las cuales pueden verse obligadas a realizar actividades de muy diverso tipo, tanto dentro del territorio nacional español, como fuera de sus fronteras. A estas actividades, a través de las cuales las Fuerzas Armadas contribuyen a la acción del Estado, para responder a una determinada situación, con sus medios personales y materiales, se les atribuye normalmente la denominación de "operaciones militares", utilizándose la expresión "contexto operativo" para designar el entorno en el que se desarrollan. $\mathrm{Al}$ análisis y exposición del marco jurídico que sirve de sustento legal a estas operaciones militares, en sus líneas generales y con carácter meramente divulgativo, es a lo que vamos a dedicar las siguientes líneas.

Palabras clave:

Fuerzas Armadas Españolas, marco jurídico, operaciones militares.

\section{ABSTRACT:}

The Spanish Armed Forces, made up of the Army, the Navy and the Air Force, are constitutionally assigned a series of missions, in the development of which they may be forced to carry out activities of a very diverse nature, both within the

* General Auditor, Asesor Jurídico del Jefe de Estado Mayor del Ejército de Tierra (España). 
national territory. Spanish, as outside its borders. These activities, through which the Armed Forces contribute to the action of the State, to respond to a certain situation, with their personal and material means, are normally attributed the name of "military operations", using the expression "context operational" to designate the environment in which they are developed. The following lines are going to be devoted to the analysis and exposition of the legal framework that serves as the legal basis for these military operations, in its general lines and with a merely informative character.

\section{Keywords:}

Spanish armed forces, legal framework, military operations.

\section{INTRODUCCIÓN}

Las Fuerzas Armadas españolas, constituidas por el Ejército de Tierra, la Armada y el Ejército del Aire, tienen constitucionalmente asignadas una serie de misiones, en el desarrollo de las cuales pueden verse obligadas a realizar actividades de muy diverso tipo, tanto dentro del territorio nacional español, como fuera de sus fronteras. A estas actividades, a través de las cuales las Fuerzas Armadas contribuyen a la acción del Estado, para responder a una determinada situación, con sus medios personales y materiales, se les atribuye normalmente la denominación de "operaciones militares", utilizándose la expresión "contexto operativo" para designar el entorno en el que se desarrollan. Al análisis y exposición del marco jurídico que sirve de sustento legal a estas operaciones militares, en sus líneas generales y con carácter meramente divulgativo, es a lo que vamos a dedicar las siguientes líneas.

\section{UN MARCO JURÍDICO PLURAL}

Las operaciones militares de las Fuerzas Armadas españolas pueden tener lugar en contextos operativos muy variados y tanto dentro como fuera de España. Esta circunstancia implica que las normas aplicables a las distintas operaciones puedan no ser idénticas. Ahora bien, en todas ellas podemos afirmar que existe un fondo o base común, constituido por la Norma Fundamental del Estado, que es la Constitución, complementado con las normas jurídicas que la desarrollan. Dentro de éstas revisten mayor relevancia, a los efectos de este estudio y como es lógico, las que tienen por objeto de regulación específico el ámbito militar. Pero al lado de las normas jurídicas que integran el Derecho nacional, las Fuerzas Armadas españolas deben respetar también todas aquellas normas internacionales que hayan pasado a formar parte de su ordenamiento jurídico, por vía convencional o consuetudinaria, normas internacionales que tienen relevancia especialmente cuando las tropas españolas se encuentran desempeñando misiones u operaciones internacionales o multinacionales, aunque no exclusivamente en ellas (piénsese, por citar solo un ejemplo, en los artículos 82 y 83 del Primer Protocolo Adicional a los Convenios de Ginebra de 1949, que imponen a los Estados 
partes la obligación de que, en todo tiempo, dispongan de asesores jurídicos que presten asesoramiento a los comandantes militares en relación con la aplicación del Protocolo y de los Convenios y respecto a la enseñanza de estas materias en las Fuerzas Armadas (art. 82) y que obliga a los Estados a difundir lo más ampliamente posible, ya en tiempo de paz, el contenido de los Convenios y del Protocolo y de incorporar su estudio en los programas de instrucción militar (art. 83) ). Todo ello hace que el marco jurídico de las Fuerzas Armadas en contextos operativos sea un cuadro jurídico plural y, en este sentido, podríamos hablar de cinco marcos que se combinan y complementan:

- El marco jurídico constitucional.

- El marco jurídico que podríamos llamar "general" (integrado por las normas jurídicas internas que desarrollan la Constitución y a las que están sujetas las Fuerzas Armadas, como cualquier otra rama de la Administración General del Estado).

- El marco jurídico penal y disciplinario, integrado por las normas penales, procesales, orgánicas judiciales y disciplinarias militares que encuadran el ejercicio de la jurisdicción militar y que otorgan a los mandos militares los instrumentos necesarios para el mantenimiento de la disciplina, con respeto a los principios constitucionales.

- El marco jurídico excepcional, que contiene las normas aplicables a las situaciones de excepción, igualmente con base en las previsiones constitucionalmente establecidas.

- Y el marco jurídico internacional, es decir, el constituido por las normas de Derecho internacional que resulten aplicables a la operación de que se trate.

Analizaremos brevemente cada uno de ellos, comenzando por el mencionado en primer lugar, el marco constitucional.

El marco constitucional.- La Constitución española, fruto de un proceso constituyente que culminó la transición política iniciada tras la muerte del General Franco y que, aprobada por las Cortes en sesión plenaria de sus dos Cámaras, fue ratificada por el pueblo español en referéndum celebrado el día 6 de diciembre de 1978, contiene varios preceptos que conviene resaltar por la importancia que tienen en relación con las Fuerzas Armadas.

Forzoso es comenzar aludiendo a su artículo 8, que define cual es la composición de las Fuerzas Armadas, señalando que están constituidas por el Ejército de Tierra, la Armada y el Ejército del Aire. La Guardia Civil, ${ }^{1}$ por lo tanto, no forma parte actualmente de las Fuerzas Armadas, aunque es un Instituto Armado

1 La Guardia Civil es un Instituto armado de naturaleza militar creado por Real Decreto de 28 de marzo de 1844. Su fundador y primer jefe fue don Francisco Javier Girón y Ezpeleta, segundo Duque de Ahumada, quien, como curiosidad histórica, cuenta al Emperador azteca Moctezuma entre sus antepasados. En efecto, un nieto de Moctezuma, bautizado y convertido al cristianismo con el nombre de Diego Luis de Moctezuma, casó en España con Francisca de la Cueva y Valenzuela, noble española, entre cuya descendencia nacería, tras varias generaciones, el segundo Duque de Ahumada. 
de naturaleza militar. Esa condición militar, de la que gozan todos sus miembros, le viene atribuida, como veremos, por las leyes que la regulan y ha sido reconocida y confirmada por reiterada doctrina jurisprudencial. Por el contrario, sí forman parte de las Fuerzas Armadas los llamados "Cuerpos Comunes" (Cuerpo Jurídico Militar, Cuerpo de Sanidad Militar, Cuerpo de Intervención y Cuerpo de Músicas Militares), a pesar de que no son parte integrante de ninguna de las tres ramas de las Fuerzas Armadas (FAS en lo sucesivo) y que reciben esa denominación para diferenciarlos de los llamados "Cuerpos específicos" de los Ejércitos.

El artículo 8 continúa precisando cuales son las misiones constitucionales atribuidas a las FAS: "garantizar la soberanía e independencia de España, defender su integridad territorial y el ordenamiento constitucional”. Es pues un precepto fundamental y el único de todo el texto constitucional que está dedicado exclusivamente a las Fuerzas Armadas.

Pero al lado de este esencial precepto, merecen nuestra atención otras disposiciones constitucionales, como el artículo 9, que impone a los militares, como a cualquier otro ciudadano, y a las Fuerzas Armadas, como a todo poder público, la obligación de someterse a la Constitución y al resto del ordenamiento jurídico y de respetar la ley en todas sus actuaciones; el artículo 62, que atribuye al Rey el mando supremo de las Fuerzas Armadas; y el artículo 97, que otorga expresamente al Gobierno de la Nación la potestad de dirigir la política interior y exterior del Estado, la Administración civil y militar y la defensa del Estado, precepto que, a su vez, es necesario poner en relación con el artículo 66.2, que atribuye a las Cortes la función de controlar la acción del Gobierno en todos los ámbitos y, por lo tanto, también en el campo militar.

Acabaremos haciendo referencia a otros dos preceptos de suma importancia: el artículo 149.1.4 ${ }^{\circ}$, que reserva al Estado la competencia exclusiva sobre Defensa y Fuerzas Armadas, negando cualquier posibilidad de que las Comunidades Autónomas, es decir las diferentes entidades territoriales que integran el Estado español, asuman competencias en la materia. Y el artículo 117.5 que proporciona un respaldo constitucional expreso a la existencia y funcionamiento de la jurisdicción militar al proclamar que "la ley regulará el ejercicio de la jurisdicción militar en el ámbito estrictamente castrense y en los supuestos de estado de sitio, de acuerdo con los principios de la Constitución", ello sin perjuicio del principio de unidad jurisdiccional, que es la base de la organización y del funcionamiento de los Tribunales. En aplicación de este principio de unidad jurisdiccional, la jurisdicción militar forma parte del poder judicial del Estado y en su cúspide se encuentra el Tribunal Supremo, al igual que ocurre con el resto de órdenes jurisdiccionales. La Sala a la que se encomienda el conocimiento de los asuntos penales militares y de los contencioso-disciplinarios militares (recursos contra sanciones disciplinarias impuestas por los mandos militares) es concretamente la Sala V, de lo Militar. Como es lógico, dada la naturaleza militar de la Guardia Civil, sus miembros están igualmente sujetos a 
la jurisdicción militar, en los términos fijados por el Código Penal Militar y por la Ley Orgánica de Régimen Disciplinario de la Guardia Civil. ${ }^{2}$

El marco jurídico general.- El que hemos dado en denominar "marco jurídico general" está constituido en realidad por el conjunto de normas que conforman el ordenamiento jurídico español, ordenamiento que deben respetar las Fuerzas Armadas como parte de la Administración General del Estado. Pero dentro de este conjunto de normas es necesario destacar aquellas disposiciones cuyo objeto de regulación específico es precisamente el ámbito militar. Mencionaremos solo las principales:

- Ley Orgánica 5/2005, de 17 de noviembre, de la Defensa Nacional. Texto normativo de importancia capital para las Fuerzas Armadas. No solo establece las bases de la organización militar, conforme a los principios contenidos en la Constitución, definiendo las atribuciones de los poderes del Estado en el ámbito de la Defensa, sino que dedica todo un Título (el Título III), a las misiones de las Fuerzas Armadas y a su control parlamentario, cuestión ésta a la que nos referiremos con más detalle dentro de un momento. Por lo que hace a la organización de las Fuerzas Armadas, el artículo 11 de la Ley establece que éstas se organizan en dos estructuras, una orgánica, para la preparación de la fuerza, y otra operativa, para su empleo en las misiones que se le asignen. Esta estructura operativa tiene por finalidad facilitar la acción conjunta de los tres Ejércitos y la acción combinada con las Fuerzas Armadas de otras naciones amigas o aliadas. Es importante mencionar, en este sentido, que el mando de la estructura operativa de las Fuerzas Armadas y la conducción estratégica de las operaciones militares corresponden, y así lo proclama el artículo 12 de la Ley, al Jefe del Estado Mayor de la Defensa, bajo la dependencia siempre del Ministro de Defensa.

Es interesante destacar igualmente que esta Ley Orgánica se refiere también a los órganos de la jurisdicción militar, recalcando que su organización y funcionamiento se basa en el principio de unidad jurisdiccional y que forman parte integrante del Poder Judicial, limitando su actuación al ámbito estrictamente castrense, de acuerdo con lo dispuesto en las leyes penales, procesales y disciplinarias militares. Por último, merecen mencionarse también los preceptos que dedica la Ley a la Guardia Civil, a la que su artículo 23 define como un Instituto armado de naturaleza militar, dependiente del Ministerio del Interior en el desempeño de sus

2 El Código Penal Militar actualmente vigente es el aprobado por la Ley Orgánica 14/2015, de 4 de octubre. El régimen disciplinario de las Fuerzas Armadas se encuentra regulado en la Ley Orgánica 8/2014, de 4 de diciembre, mientras que es la Ley Orgánica 12/2007, de 22 de octubre, la que contiene la regulación del régimen disciplinario aplicable a la Guardia Civil. Interesa mencionar que según el artículo 81 de la Constitución son leyes orgánicas las relativas al desarrollo de los derechos fundamentales y libertades públicas, las que aprueban los Estatutos de Autonomía, el régimen electoral general y las demás previstas en la Constitución. Por su importancia, su aprobación, modificación o derogación exige mayoría absoluta del Congreso, en una votación final sobre el conjunto del proyecto. 
funciones militares, y del Ministerio de Defensa en el cumplimiento de las misiones militares que se le encomienden. Estas misiones militares que se pueden encomendar a los miembros de la Guardia Civil se encuentran desarrolladas en el Real Decreto 1438/2010, de 5 de noviembre. El carácter militar de la Guardia Civil es, por otro lado, lo que justifica que sus miembros estén igualmente sujetos a las disposiciones de las Reales Ordenanzas para las Fuerzas Armadas, salvo en lo que se opongan a lo previsto en su legislación específica.

- Ley 36/2015, de 28 de septiembre, de Seguridad Nacional, que es preciso traer a colación, por cuanto la defensa de España y la acción del Estado para contribuir a la seguridad internacional forman parte del concepto de seguridad nacional que constituye el objeto de regulación de este texto normativo. La Ley de Seguridad Nacional configura a la Defensa Nacional como uno de los componentes esenciales de la Seguridad Nacional, y en el sistema de seguridad que prevé otorga un papel preponderante, tanto al Ministro de Defensa como al Jefe del Estado Mayor de la Defensa, de forma coherente con la regulación contenida en la Ley Orgánica de la Defensa Nacional.

- Ley Orgánica 9/2011, de 28 de julio, de derechos y deberes de los miembros de las Fuerzas Armadas. Los miembros de las Fuerzas Armadas son, como cualquier otro ciudadano, titulares de los derechos fundamentales y de las libertades públicas constitucionalmente reconocidas, aunque son susceptibles de algunas limitaciones que vienen exigidas por la necesidad de garantizar el mantenimiento de la disciplina, el orden jerárquico y la disponibilidad permanente para el servicio, pilares en los que se asienta el correcto funcionamiento de las Fuerzas Armadas. Esta ley recoge esas limitaciones y proclama al mismo tiempo las reglas esenciales del comportamiento del militar, reglas que venían ya formuladas y desarrolladas en las Reales Ordenanzas para las Fuerzas Armadas, de rango reglamentario, aprobadas por el Real Decreto 96/2009, de 6 de febrero.

- Ley 39/2007, de 19 de noviembre, de la Carrera Militar, norma que tiene por objeto regular el régimen del personal militar profesional y todos aquellos aspectos que lo conforman.

Marco jurídico penal y disciplinario.- Como hemos tenido ya ocasión de exponer, la Constitución española en su artículo 117 alude de forma expresa a la jurisdicción militar, limitándola al ámbito estrictamente castrense, pero integrándola con el resto de órdenes judiciales bajo el principio de unidad jurisdiccional. La organización y el ejercicio de la jurisdicción militar encuentra su desarrollo en las siguientes leyes:

- Ley Orgánica 4/1987, de 15 de julio, de Organización y Competencia de la Jurisdicción Militar que, como su propio título indica, regula la organización, composición y competencias de los distintos Juzgados y Tribunales que integran la jurisdicción militar, conteniendo igualmente 
normas dedicadas a los Secretarios Relatores, a la Fiscalía Jurídica Militar y a los letrados que intervienen en los procedimientos militares. Simplemente volveremos a recordar que, en aplicación del principio de unidad jurisdiccional, en la cúspide de la organización judicial militar se encuentra la Sala V del Tribunal Supremo (Sala de lo Militar), a quién compete, por vía del recurso de casación, la revisión de las decisiones adoptadas, tanto por el Tribunal Militar Central como por los cinco Tribunales Militares Territoriales existentes, en los casos previstos por esta misma Ley y por la Ley Procesal Militar.

- Ley Orgánica 2/1989, de 13 de abril, Procesal Militar, que contiene normas detalladas sobre el régimen de Juzgados y Tribunales militares, cuestiones de competencia y reglas aplicables a la iniciación, sustanciación y fallo de los procedimientos judiciales, así como a la ejecución de las resoluciones judiciales y recursos que se pueden interponer contra ellas.

- Ley Orgánica 14/2015, de 14 de octubre, del Código Penal Militar, de reciente promulgación, que ha venido a sustituir al anterior Código Penal Militar de 9 de diciembre de 1985 (L.O. 13/85), para modernizarlo, adaptándolo a las trasformaciones sufridas por las Fuerzas Armadas (incorporación de la mujer y participación frecuente en operaciones internacionales, entre otras) y reduciendo su articulado al darle un mayor carácter complementario del Código Penal Común.

- Y finalmente, mencionaremos en este apartado la Ley Orgánica 8/2014, de 4 de diciembre, de Régimen Disciplinario de las Fuerzas Armadas, de reciente entrada en vigor, en sustitución de la anterior del año 1998, modificada básicamente por los mismos motivos que han llevado a la actualización del Código Penal Militar. Como novedad importante de la Ley actual, se introducen las sanciones económicas para las infracciones cometidas por los militares, al lado de la posibilidad de imponer sanciones restrictivas (faltas leves) o privativas (faltas graves y muy graves) de libertad, aunque reduciendo sensiblemente su duración con respecto a la normativa anterior; y se dedica un capítulo específico a las Unidades y personal destacado en zona de operaciones. Debemos igualmente mencionar que las sanciones impuestas por los mandos militares son susceptibles de ser revisadas por los Tribunales integrantes de la jurisdicción militar, a través de los recursos contenciosos-disciplinarios militar ordinario y preferente y sumario. Para acceder a ellos es preciso, no obstante, haber agotado previamente los recursos administrativos que se pueden interponer ante la cadena de mando. ${ }^{3}$

Finalmente diremos que a los miembros de la Guardia Civil, pese a estar sujetos a un régimen disciplinario propio, constituido por la Ley

Salvo en el caso del recurso contencioso disciplinario preferente y sumario contra sanciones por faltas graves y muy graves, en el que ese requisito no es necesario, por disponerlo así el artículo 518 b) de la Ley Orgánica Procesal Militar. 
Orgánica 12/2007, de 22 de octubre, les es aplicable el régimen disciplinario de las Fuerzas Armadas cuando actúen en misiones de carácter militar o integrados en unidades militares, por establecerlo así la Disposición Adicional Quinta de la Ley Orgánica de Régimen Disciplinario de las Fuerzas Armadas, de conformidad con lo previsto en la Disposición Adicional Sexta de la propia Ley de Régimen Disciplinario de la Guardia Civil.

Marco excepcional.- El que calificamos de marco jurídico excepcional es el constituido por la Ley Orgánica 4/1981, de 1 de junio, reguladora de los estados de alarma, excepción y sitio. En efecto, esta Ley tiene su relevancia en el ámbito de las Fuerzas Armadas, particularmente en los supuestos en los que sea declarado el estado de sitio, en los que, conforme a sus artículos 33 y 35, se podrán otorgar por parte del Gobierno facultades extraordinarias a la Autoridad militar, para hacer cumplir las decisiones gubernamentales y, por otro lado, el Congreso de los Diputados podrá también ampliar la competencia de la Jurisdicción militar, decidiendo qué delitos quedan sometidos a ella durante la vigencia del estado de sitio.

Marco internacional.- Finalmente, y de gran importancia en relación con las operaciones de las Fuerzas Armadas fuera de las fronteras nacionales, a las que nos referiremos con más detalle posteriormente, es el conjunto de normas internacionales, tanto de derecho consuetudinario como convencional, que vinculan a España y que forman parte por ello de su ordenamiento jurídico. Mencionaremos en particular la Carta de Naciones Unidas, todas las normas que integran el Derecho Internacional Humanitario, las normas que forman parte del Derecho Internacional de los Derechos Humanos y los Acuerdos relativos al Estatuto de fuerzas ratificados por España o suscritos por las Organizaciones internacionales en cuyas misiones participan fuerzas españolas.

\section{LAS MISIONES DE LAS FUERZAS ARMADAS A LA LUZ DE LA CONSTITUCIÓN Y DE LA LEY ORGÁNICA DE LA DEFENSA NACIONAL}

Expuesto ya, en líneas generales, el marco jurídico plural en el que se encuadra la actividad de las Fuerzas Armadas españolas, pasaremos ahora a analizar de una forma algo más concreta las misiones que el ordenamiento jurídico les asigna y los aspectos más relevantes de su regulación.

Como ya ha quedado dicho, la Constitución asigna a las Fuerzas Armadas, en su artículo 8, la esencial misión de la defensa de la soberanía e independencia de España, por un lado; y la de defensa de su integridad territorial y del ordenamiento constitucional, por otro. Cuatro misiones básicas, por tanto, que se reiteran y concretan en la Ley Orgánica de la Defensa Nacional. El artículo 15 de este texto normativo, en efecto, comienza recordando las misiones constitucionalmente atribuidas a las Fuerzas Armadas, para a continuación precisar como una de sus 
funciones propias, y de forma completamente lógica, la contribución militar a la defensa de España y, lógicamente, de los españoles, incluyendo expresamente (art. 15.4) la posibilidad de llevar a cabo misiones de evacuación de españoles residentes en el extranjero, cuando las circunstancias de inestabilidad en un país pongan en peligro su vida o sus intereses. Pero además, de acuerdo con una concepción amplia y moderna de la defensa y de la seguridad nacionales, que no se limita a la estricta defensa territorial de las fronteras patrias, sino que tiene en cuenta la importancia de mantener la paz y la seguridad internacionales, como un medio para garantizar la seguridad nacional, dado el carácter global de los problemas del mundo actual, el precepto añade que las Fuerzas Armadas españolas deben contribuir militarmente a la defensa de sus aliados, en el marco de las organizaciones internacionales de las que España forma parte, y les encomienda también la función de contribuir al mantenimiento de la paz y de la estabilidad internacionales y de participar en misiones de ayuda humanitaria. Se otorga, pues, a las Fuerzas Armadas una dimensión internacional clara, orientada en un doble sentido, la defensa colectiva en unión de los países aliados frente a eventuales agresiones, y la participación en misiones de paz, de estabilización y ayuda humanitaria, en aras del mantenimiento de la paz y de la seguridad internacionales.

Al lado de esa dimensión internacional, se contempla también una dimensión interna pues el precepto impone a las Fuerzas Armadas la obligación de preservar, en unión del resto de Instituciones del Estado, el bienestar y la seguridad de los ciudadanos en los supuestos de grave riesgo, calamidad, catástrofes u otras necesidades públicas, de acuerdo con la legislación vigente. Quiere ello decir que las Fuerzas Armadas tienen la obligación de intervenir, conforme a los procedimientos legalmente previstos, en apoyo de los servicios de protección civil o de las Fuerzas y Cuerpos de Seguridad, en todo el amplio abanico de situaciones que pueden poner en peligro la seguridad de los ciudadanos, sean provocadas por fenómenos naturales o no. En relación con los primeros, merece la pena mencionar la creación de una Unidad, desplegada en todo el territorio nacional, que tiene la misión específica de intervenir en este tipo de situaciones. Es la Unidad Militar de Emergencias (UME), constituida en el mes de octubre de 2005 y a la que su buen hacer desde el momento mismo de su creación le ha granjeado un prestigio y aprecio creciente entre la población y las instituciones locales y regionales.

Estas misiones genéricas atribuidas a las Fuerzas Armadas se concretan en una multiplicidad de operaciones, tanto dentro de España como en el exterior, que abarcan todo el espectro de situaciones posibles. La Ley Orgánica de la Defensa Nacional (LODN) menciona en su artículo 16, sin carácter exhaustivo, algunas de ellas, en particular, las operaciones de vigilancia de los espacios marítimos y control del espacio aéreo, el apoyo a las Fuerzas y Cuerpos de Seguridad del Estado en la lucha contra el terrorismo y a las instituciones y organismos responsables de los servicios de rescate, en las tareas de búsqueda y salvamento así como la colaboración en operaciones de mantenimiento de la paz y estabilización internacional. 
A estas últimas nos referiremos con más detenimiento, puesto que implican la participación de tropas españolas fuera de las fronteras nacionales y debemos comenzar señalando que la LODN regula este tipo de operaciones introduciendo dos novedades importantes: exigiendo, por un lado, el cumplimiento de un requisito nuevo, inexistente con anterioridad a su entrada en vigor (la necesidad de autorización previa por parte del Congreso de los Diputados, salvo casos excepcionales); y estableciendo, por otro lado, las condiciones precisas que deben concurrir "sine qua non", para que el Congreso pueda dar esa autorización.

Efectivamente, como ha tenido ocasión de poner de manifiesto Vicente Moret Millás, ${ }^{4}$ hasta la promulgación y entrada en vigor de la LODN no existía ninguna disposición que articulase de forma concreta la intervención del Parlamento en la adopción de una decisión tan importante como es la participación de tropas españolas en misiones fuera de nuestras fronteras nacionales. Hasta entonces, la decisión la tomaba el Gobierno en el ejercicio de sus atribuciones constitucionales otorgadas por el artículo 97 anteriormente mencionado (dirección de la política interior y exterior y de la defensa del Estado), limitándose las Cortes a realizar un control "a posteriori", a través de los mecanismos de control habituales (preguntas parlamentarias, petición de comparecencia de miembros del Gobierno...). La LODN ha venido a poner fin a esa situación, estableciendo su artículo 4.2 que al Congreso de los Diputados le corresponde autorizar, con carácter previo, la participación de las Fuerzas Armadas en misiones fuera del territorio nacional. De acuerdo con ello, el artículo 17 de la Ley regula las distintas modalidades de autorización previendo un triple procedimiento, un procedimiento que podríamos calificar de "ordinario" (art. 17.1), un procedimiento de "urgencia" (art. 17.2) y un procedimiento de "máxima urgencia" (art. 17.3)5 El procedimiento ordinario es el previsto en el apartado 1 del artículo 17, según el cual, "para ordenar operaciones en el exterior que no estén directamente relacionadas con la defensa de España o del interés nacional, el Gobierno realizará una consulta previa y recabará la autorización del Congreso de los Diputados" (art. 17.1). Por lo tanto, se impone la obligación general al Gobierno de recabar esa autorización antes de decidir la participación de soldados españoles fuera de las fronteras nacionales. Interesa subrayar, no obstante, que esa obligación puede ser objeto de excepción en los casos que menciona el precepto: que la operación de que se trate esté directamente relacionada con la defensa de España o con la defensa del interés nacional. Como es posible apreciar, la formulación de la excepción es tremendamente genérica, de manera que podría dar lugar a una interpretación muy amplia de lo que se debe entender tanto por "relación directa" con la defensa o con el interés nacional, como sobre lo que se debe incluir en los propios conceptos de "defensa de España" y de "interés nacional", conceptos éstos que pueden

4 MORET MILLÁS, Vicente: "La autorización de las operaciones en el exterior de las Fuerzas Armadas: una propuesta de regulación", en CEFlegal Revista Práctica de Derecho. Comentarios y casos prácticos, números 151-152, 2013.

5 Sigo la denominación que a tales modalidades de autorización da Vicente Moret en el artículo citado en la nota 4 anterior. 
ser objeto de interpretaciones muy estrictas o, por el contrario, muy extensas. En cualquier caso, de momento el problema no se ha llegado a plantear y el Gobierno ha venido cumpliendo con normalidad con la obligación impuesta por el artículo 17.1, sin haber recurrido a las posibles excepciones, aunque bien es verdad que siguiendo procedimientos diversos (unas veces la autorización la ha concedido el Pleno del Congreso, mientras que otras la autorización ha sido concedido por la Comisión de Defensa).

Por lo que respecta al procedimiento de urgencia, lo contempla el apartado 2 del artículo 17, a cuyo tenor las misiones en el exterior que, de acuerdo con los compromisos internacionales asumidos por España, requieran una respuesta rápida o inmediata para hacer frente a determinadas situaciones, se harán por procedimientos de urgencia que permitan cumplir con los compromisos asumidos. Esos procedimientos de urgencia que prevé la Ley, sin embargo, no han sido todavía regulados.

Finalmente, el procedimiento al que hemos denominado de "máxima urgencia” es el que prevé el artículo 17.3, que autoriza al Gobierno a actuar y tomar la decisión del envío de tropas al exterior, sin la previa autorización del Congreso, pero solo en aquellos casos en los que la urgencia sea tan grande que no dé tiempo a solicitarla, debiendo en tales casos someter la decisión lo antes posible al Congreso para su ratificación.

En cuanto a las condiciones necesarias para que el Congreso pueda conceder esa autorización para el despliegue en el exterior (probablemente para evitar que se vuelvan a producir en el futuro intervenciones cuya conformidad al Derecho internacional ha sido discutida, como es particularmente la participación de fuerzas españolas en la operación "Allied Force", llevada a cabo por la OTAN en Kosovo en el año 1999), el legislador ha fijado en el artículo 19 de la Ley una serie de requisitos que garantizan que la autorización parlamentaria no pueda ser otorgada si no se verifica que la operación en la que vayan a participar los soldados españoles es plenamente conforme a las normas de Derecho internacional. Concretamente, el precepto citado establece que las misiones de las Fuerzas Armadas en el exterior deben estar justificadas por alguno de los siguientes motivos:

- Que se realicen a petición expresa del Gobierno del Estado en cuyo territorio se vaya a desarrollar la misión.

- Que estén oportunamente autorizadas por Resoluciones del Consejo de Seguridad de las Naciones Unidas.

- O que hayan sido acordadas por organizaciones internacionales de las que España forme parte, particularmente la Unión Europea o la Organización del Tratado del Atlántico Norte, en el marco de sus respectivas competencias.

A parte de ello, el precepto especifica que las misiones asignadas a las Fuerzas Armadas españolas deben: 
- Cumplir con los fines defensivos, humanitarios, de estabilización o mantenimiento y preservación de la paz, previstos y ordenados, en su caso, por las mencionadas organizaciones.

- Y que, en todo caso, y este es un requisito esencial, las misiones deben ser conformes con la Carta de las Naciones Unidas y no deben contradecir ni vulnerar los principios de Derecho internacional convencional que España ha incorporado a su ordenamiento, de acuerdo con la Constitución.

\section{MARCO JURÍDICO APLICABLE A LOS DISTINTOS TIPOS DE MISIÓN}

Una vez expuestas las líneas que enmarcan el régimen jurídico aplicable a las misiones de las Fuerzas Armadas con carácter general, podemos ya aludir al marco aplicable en los diferentes contextos operativos en que pueden ser desarrolladas esas misiones. La normativa que será de aplicación en cada caso vendrá determinada por el contexto operativo en que se desarrolle la misión, aunque una gran parte de las normas que integran el marco jurídico plural de las Fuerzas Armadas y que hemos mencionado más arriba acompañan al militar español allá donde se desplace y cualquiera que sea el tipo de misión que desempeñe. Las normas relativas al régimen de personal, o las normas disciplinarias o penales militares, por ejemplo, se aplican al personal militar español donde quiera que se encuentren y con independencia de la misión en que estén participando. Sin embargo, el régimen jurídico aplicable a la operación en la que participan las Fuerzas Armadas españolas puede variar según el contexto operativo en el que se encuentren desplegadas. Y en este sentido, podemos distinguir, de una forma muy general, los siguientes tipos de operaciones:

a. Operaciones de combate

Las Fuerzas Armadas españolas pueden verse obligadas a realizar misiones $\mathrm{u}$ operaciones de combate en el ejercicio del derecho de legítima defensa individual o colectiva, reconocido en el artículo 51 de la Carta de Naciones Unidas, frente a un ataque o agresión armada; pero también cuando sea necesario usar la fuerza en cumplimiento y con el amparo de una resolución del Consejo de Seguridad de Naciones Unidas adoptada en el marco del Capítulo VII de la Carta de Naciones Unidas. Cuando esto ocurra, dando lugar a la participación de tropas españolas en un conflicto armado, la conducta de las Fuerzas Armadas españolas debe acomodarse y respetar, ante todo, las normas del Derecho Internacional Humanitario (DIH). En este sentido hay que señalar que España es parte de los Convenios de Ginebra de 1949, de sus Protocolos Adicionales y de todos los Convenios y Acuerdos internacionales que constituyen el núcleo esencial del Derecho Internacional Humanitario. Y que los artículos 608 y siguientes del Código Penal común, aplicables al personal militar por disponerlo así el artículo 9 del Código Penal Militar tipifica los delitos contra las personas y bienes protegidos en caso de conflicto armado. Por otro lado, los principios del Derecho internacional Humanitario deben regir también la actuación de las Fuerzas Armadas españolas 
en caso de verse involucradas en acciones de combate en el curso de operaciones de paz o de gestión de crisis. Por último hay que precisar que la aplicación preferente del Derecho Internacional Humanitario no excluye la aplicación del Derecho Internacional de los Derechos Humanos, que deberá ser igualmente tenido en cuenta como norma de actuación en aquellos aspectos no regulados de manera específica por el DIH.

\section{b. Operaciones de paz}

Las operaciones militares que se pueden emprender con la finalidad de mantener la paz y la seguridad internacionales son de una variedad enorme. No existe unanimidad, sobre la terminología para designarlas ni sobre su definición precisa. Una clasificación muy extendida se basa en la diferenciación efectuada en el marco de las Naciones Unidas, por parte de su Secretario General, que refleja la evolución sufrida por las clásicas Operaciones de Mantenimiento de la Paz (OMPs), que han pasado de tener funciones meramente de observación o de interposición entre contendientes a tener un carácter complejo y multifuncional. El documento " Un Programa de Paz" (Informe del Secretario General de Naciones Unidas, A/47/277-S 24111, 17 junio 1992), complementado por el Suplemento al Programa de Paz (A/50/60 -S/1995/1), de 25 de enero de 1995, distingue entre diplomacia preventiva (conflict prevention), medidas de establecimiento de la paz (peacemaking), operaciones de mantenimiento de la paz (peacekeeping), medidas de imposición de la paz (peace-enforcement), medidas de consolidación de la paz (peacebuilding) y ayuda humanitaria (humanitarian assistance). En cualquier caso, aquí nos vamos a referir a ellas con la denominación genérica de "operaciones de paz", término que permite englobar todas ellas, así como las operaciones que se mencionan en la Ley Orgánica de Defensa Nacional.

El régimen jurídico de estas operaciones depende, como es lógico, del tipo de misión de que se trate. Si nos encontramos ante una misión desplegada en el territorio de un Estado a petición del Gobierno de éste, habrá que estar en primer lugar a los acuerdos alcanzados con el Estado en el que se desarrolla la misión (al que se suele dar el nombre de "Estado anfitrión" o "Estado receptor") que normalmente imponen como primera obligación de las fuerzas extranjeras, la de respetar las normas nacionales locales. A parte de ellas, será necesario observar igualmente las normas de Derecho internacional que resulten aplicables; y entre ellas ocupan un lugar esencial las normas que integran el llamado Derecho Internacional de los Derechos Humanos. En este sentido conviene ya adelantar que España es parte del Convenio Europeo de Derechos Humanos y que según reiterada jurisprudencia del Tribunal Europeo de Derechos Humanos, las obligaciones asumidas en virtud de este Convenio tienen eficacia extraterritorial, de manera que sus normas deben ser respetadas con respecto a toda persona que se encuentra, de una u otra forma, bajo la jurisdicción de las fuerzas españolas, aun fuera del territorio español. Un ejemplo de misión desempeñada por las tropas españolas a petición del Gobierno de otro Estado es la desarrollada actualmente en Irak, misión limitada al adiestramiento de soldados iraquíes, aun cuando se trata de una 
misión encuadrada en una operación más amplia, llevada a cabo por una coalición de Estados, dentro de los esfuerzos por combatir al denominado Estado Islámico.

Si se trata de otro tipo de operaciones, en las que el despliegue de las Fuerzas Armadas españolas se produce como consecuencia de un mandato o decisión de una Organización Internacional, el régimen jurídico aplicable a los soldados españoles en el territorio donde se hallen desplegados viene determinado, en primer lugar, por el propio mandato de la misión. La situación más habitual, aun cuando la misión se desarrolle en el marco de una Organización Internacional de las que España forma parte, como la OTAN o la Unión Europea (UE), es que el mandato esté configurado por una resolución del Consejo de Seguridad de Naciones Unidas que autorice el despliegue en el territorio de un Estado determinado, normalmente con el acuerdo del propio Estado afectado, aunque hay ocasiones en las que esa circunstancia puede no darse. El mandato fija no sólo los objetivos de la operación, sino también las bases legales para el uso de la fuerza durante su desarrollo, estableciendo al propio tiempo los límites a dicho uso. El régimen jurídico se complementa con el acuerdo sobre el estatuto de fuerzas (conocido corrientemente con la abreviatura "SOFA", acrónimo de los términos anglosajones "Status of Forces Agreement"). Hay que recordar que en las operaciones de mantenimiento de la paz llevadas a cabo en el marco de la Organización de Naciones Unidas, los tropas que forman parte de la misión, a título de observadores o de contingentes, gozan de la protección otorgada por el Convenio sobre la Seguridad del Personal de las Naciones Unidas y Personal Asociado, de 9 de diciembre de 1994. En este tipo de operaciones juega igualmente un papel fundamental el respeto por las tropas intervinientes, sean españolas o no, de las normas del Derecho Internacional de los Derechos Humanos, que se aplican con prevalencia al Derecho Internacional Humanitario. Éste sólo sería de aplicación, de acuerdo con lo proclamado por el Boletín del Secretario General sobre Observancia del Derecho Internacional Humanitario por las Fuerzas de Naciones Unidas, de 6 de agosto de $1999^{6}$ y conforme a la opinión de la mayor parte de la doctrina, en caso de que las fuerzas de paz, por un deterioro de la situación, se vieran envueltas en acciones de combate que por sus características de intensidad y duración, se pudieran equiparar a una situación de conflicto armado y solo mientras dure esa situación. Sería igualmente de aplicación en el caso de operaciones coercitivas o de imposición de la paz, si dieran lugar a una situación de conflicto armado.

c. Otras operaciones de gestión de crisis (lucha contra la piratería, contra el tráfico de personas, etcétera.)

Finalmente mencionaremos otro tipo de operaciones en las que el régimen jurídico aplicable viene determinado por un conjunto de normas que hay que poner en estrecha relación. Es el caso, por ejemplo, de las operaciones de lucha contra la piratería o contra el tráfico o trata de personas, donde aparte del mandato de la misión, si ésta se desarrolla en el marco de una organización internacional (como es el caso de la operación Atalanta, operación de lucha contra la piratería en el

6 Boletín del Secretario General, ST/SGB/1999/13, de 6 de agosto de 1999. 
Índico, liderada por la UE; o de la operación Sophia, de lucha contra las mafias de inmigración en el Mediterráneo, también llevada a cabo por efectivos de varias naciones en el marco de la UE) hay que tener muy presente tanto el Derecho nacional español como el Derecho internacional general aplicable a la operación.

Resta aludir a dos puntos importantes. En todas las operaciones que hemos mencionado y en las que, en mayor o menor medida, es posible que sea necesario recurrir al uso de la fuerza, el empleo de ésta se regula y se limita a través de las llamadas Reglas de Enfrentamiento, también denominadas en algunos países iberoamericanos Reglas de Empeñamiento (conocidas habitualmente con el acrónimo anglosajón "ROE" - Rules of Engagement-). Las Reglas de Enfrentamiento se pueden definir como las directivas o instrucciones que el mando de una operación dirige a las fuerzas participantes en la misma con objeto de fijar las condiciones, casos y circunstancias en las que se autoriza el uso de la fuerza o la realización de acciones que pueden ser consideradas provocativas. Son en definitiva un instrumento de control de las unidades y del personal militar que participa en la misión para garantizar que las operaciones se desarrollan conforme a los objetivos políticos y militares deseados y que se respeta tanto la legalidad nacional como la internacional. Las ROE emanan de la máxima autoridad que dirige la operación y deben ser difundidas a los diferentes escalones de la cadena de mando, hasta llegar al último soldado, pero cada uno a su nivel. Habrá Reglas de Enfretamiento/Empeñamiento que sólo deban ser conocidas por los escalones superiores de la cadena de mando (el empleo de ciertos medios o métodos de combate, por ejemplo), mientras que otras (la mayor parte) deberán ser conocidas por todo el personal militar que participa en la misión. Importante señalar que las ROE dejan siempre a salvo el derecho de legítima defensa en caso de agresión o intento de agresión y que tanto en la elaboración como en la aplicación de las ROE es de una extraordinaria importancia contar con el adecuado asesoramiento jurídico.

Por otro lado, debemos recordar que como mencionábamos al exponer brevemente las líneas generales de la Ley Orgánica de la Defensa Nacional, la conducción de las operaciones de las Fuerzas Armadas españolas corresponde al Jefe del Estado Mayor de la Defensa, que cuenta para ello con la asistencia del Jefe del Mando de Operaciones. Sin embargo, cuando las operaciones se llevan a cabo en el marco de una Organización Internacional y bajo el mando del Jefe de la Fuerza designado por esta misma organización, es preciso realizar una transferencia de autoridad (TOA) para que las Fuerzas españolas puedan pasar a depender de la cadena de mando establecida por la organización para la operación. En esa transferencia de autoridad se especifican las condiciones en las que las tropas españolas quedaran bajo el mando y control de las autoridades militares de la operación, aunque conviene señalar que no suele producirse una transferencia total del mando. Las naciones mantienen siempre un cierto grado de mando y control sobre sus propias tropas. En todo caso, la aplicación del régimen disciplinario y del régimen penal queda reservado al llamado Estado de origen y así se especifica en los acuerdos que regulan el estatuto de las tropas y la participación de éstas en la misión. 


\section{RESEÑA, A TÍTULO DE EJEMPLO, DE LOS DISTINTOS TIPOS DE MISIONES EFECTUADAS POR LAS FUERZAS ARMADAS ESPAÑOLAS EN EL EXTERIOR}

Dejando de lado precedentes históricos más remotos, la participación de soldados españoles en misiones en el exterior tiene un antecedente más próximo, y poco conocido, en la intervención de médicos y enfermeros militares españoles en la guerra de Vietnam. En efecto, el Gobierno español de la época respondió afirmativamente a la petición de apoyo emitida por el Gobierno de los Estados Unidos, aunque bien es verdad que lo hizo de una forma limitada, puesto que el apoyo español no pasó del envío de una misión sanitaria, pero que estuvo desplegada en el delta del Mekong un buen número de años (de 1966 a 1971). Durante esos años, un grupo de médicos y enfermeros españoles se fueron sucediendo en varios relevos para gestionar un pequeño hospital que, a pesar de no ser muy grande, atendió tanto a militares como a civiles de la zona, dejando un grato recuerdo de su actuación.

Pero la realidad es que la participación de las Fuerzas Armadas españolas en operaciones en el exterior, tal y como se desarrollan en la actualidad, comenzó varios años más tarde y, específicamente, a partir del año 1989. Fue en ese año en el que se decidió la primera participación de militares españoles en una misión de las Organización de Naciones Unidas, concretamente en Angola (UNAVEM I), misión a la que se incorporaron 7 oficiales españoles como observadores. La participación en esta misión y en la que le sucedió (UNAVEM II) continuó varios años, hasta el mes de diciembre de 1993. A esta primera misión, limitada al envío de observadores, siguió el despliegue del primer contingente de tropas, también en el marco de Naciones Unidas y en el territorio africano, no muy lejos de donde desplegaron los observadores. Concretamente en Namibia donde, encuadrada dentro de la misión "United Nations Transition Group in Namibia (UNTAG)" se desplegó a mediados de 1989 un contingente de ocho aviones "CASA Aviocar" pertenecientes al Ala 35 del Ejército del Aire, con sus correspondientes elementos de apoyo, reforzados durante el periodo de un mes y medio por un Hércules C-130 del Ala 31, contingente que permaneció en Namibia, proporcionado apoyo aéreo a la misión, junto a una unidad de helicópteros italiana, durante el periodo de un año que duró la misión, por cierto, una de las finalizadas con mayor éxito de las efectuadas por Naciones Unidas.

Desde estas primeras participaciones, la presencia de Fuerzas españolas en varios continentes, en el marco de misiones de mantenimiento de la paz, de reconstrucción, de ayuda humanitaria, de estabilización, de intervención en casos de catástrofes naturales e incluso de imposición de la paz ha sido algo constante, tanto en el marco de las Naciones Unidas, como en el marco de otras Organizaciones como la OTAN y la UE, y en algún caso también de forma estrictamente nacional.

A título de ejemplo, podemos hacer un pequeño repaso de los distintos tipos de operaciones desarrolladas en el exterior por las FAS españolas. Aparte de las 
citadas, podemos mencionar, como ejemplo de una misión de protección de la población civil y de ayuda humanitaria, en este caso de la población kurda del Norte de Irak, la misión llevada a cabo en ese territorio por fuerzas de la Brigada Paracaidista en el año 1991, en el marco de la operación denominada "Provide Comfort". Como muestra de la participación en operaciones clásicas de mantenimiento de la paz, además de la misión en Namibia, podríamos citar la participación en UNPROFOR (United Nations Protection Force) en Bosnia-Herzegovina durante los años 1992 a 1995, año en que la participación española continuó, pero ya en el marco de la OTAN (operaciones "Stabilization Force" —SFOR - e "Implementation Force" - IFOR_); y la participación en UNIFIL (United Nations Interim Force in Lebanon), en el Líbano, comenzada en el año 2006 y que continúa en la actualidad. En esta operación España ostenta el mando de una de las Brigadas Multinacionales desplegadas sobre el terreno y un General español, el General Alberto Asarta, fue además Jefe de Misión ("Head of Mission") durante un periodo de dos años, reuniendo en su persona la condición de Jefe de la Fuerza y máxima autoridad política de la operación. Las misiones de las Naciones Unidas han contado también con una nutrida participación de oficiales españoles como observadores. Por no citar más que algunas de las desarrolladas en el continente americano podríamos mencionar la participación en ONUCA (diciembre de 1989 a diciembre de 1991) en diversos países de Centroamérica, donde fue nombrado ya por primera vez un oficial español como Jefe de la Misión.

Por lo que respecta a la participación en misiones de mantenimiento de la paz y de estabilización en el marco de la Organización del Tratado del Atlántico Norte, además de las mencionadas SFOR e ISFOR en Bosnia-Herzegovina, es preciso mencionar la participación en KFOR (Kosovo Force), a partir del año 2000 y hasta el año 2009, y la contribución aportada a ISAF (International Security Assistance Force) en Afganistán, durante los años 2003 a 2014, aunque soldados españoles fueron ya desplegados en Afganistán en el año 2002. La operación de ISAF, sustituida a principios de 2015 por la operación "Resolute Support", que cuenta también con participación española, ha obligado a los soldados españoles a verse envueltos con frecuencia en acciones de combate, por el hostigamiento sufrido de fuerzas insurgentes, y es sin duda la que ha implicado un sacrificio más elevado en número de fallecidos y heridos. No es, sin embargo, la única misión en la que las fuerzas españolas han intervenido haciendo uso de la fuerza en el marco de la OTAN. La llamada operación "Allied Force", campaña aérea llevada a cabo por decisión del Consejo Atlántico (máximo órgano de dirección política de la Organización) durante el año 1999 contra objetivos militares en Yugoslavia (sobre cuya legalidad no vamos a entrar a debatir aquí, aunque es necesario señalar que ha sido puesta en duda en algunos sectores políticos y doctrinales, por no haberse realizado al amparo de una resolución del Consejo de Seguridad de Naciones Unidas), contó también con la intervención de fuerzas aéreas españolas, al igual que la denominada operación "Unified Protector", campaña aérea efectuada contra objetivos del régimen del Presidente Gadafi en Libia en el año 2011, ésta vez sí con el pleno apoyo de la comunidad internacional, expresado a través de las 
resoluciones 1970 (2011) y 1073 (2011), de 26 de febrero y 17 de marzo de 2011, del Consejo de Seguridad de Naciones Unidas.

En el marco de los esfuerzos que lleva a cabo la Unión Europea para contribuir al mantenimiento de la paz y de la seguridad internacionales, España ha participado en muchas de las operaciones puestas en marcha, de las que mencionaremos solo, a título de ejemplo, la primera de ellas, la Operación "Concordia", en el año 2003, en la antigua República yugoslava de Macedonia, la operación "Atalanta", de lucha contra la piratería en el Indico y en el Golfo de Aden, en la que todavía se encuentra España participando, operación que ha dado unos resultados francamente positivos que han llevado a la drástica disminución (casi desaparición) de los ataques piratas en la zona. Y la misión desplegada en la actualidad en Mali (European Union Training Mission), donde un equipo de instructores de diferentes países europeos adiestran a las fuerzas armadas de Mali, con el objetivo de capacitarlas para poder enfrentarse con eficacia a las fuerzas insurgentes del Norte del país. Aun cuando no forman parte de esta misión, mencionaremos también la presencia de dos contingentes del Ejército del Aire (con base en Senegal y en Gabón), que proporcionan apoyo aéreo tanto a la EUTM Malí, como a la misión EUTM en la República Centroafricana y a las fuerzas francesas desplegadas en la operación Barkhane.

Otra misión en la que ha sido preciso recurrir al uso de la fuerza, aun cuando ha sido en el marco de una coalición internacional de Estados, y no en el marco de una Organización, ha sido la desarrollada en Irak, en los años 2003-2004. En Irak España mantiene hoy un día una presencia militar, en calidad de instructores de fuerzas regulares y de fuerzas de operaciones especiales, en el cuadro de la operación denominada "Inherent Resolve".

Y para terminar este breve repaso a los diferentes tipos de misiones desempeñados por las Fuerza Armadas españolas, mencionaremos las operaciones de asistencia y ayuda humanitaria realizadas en diversos países y continentes, con ocasión de desastres naturales (terremotos, inundaciones, etc...). Es el caso de las operaciones, puramente nacionales, llevadas a cabo en Mozambique (1994), Paquistán (2004) o Haití (2010).

Cerraremos estas líneas señalando que, al momento de escribirlas (enero de 2019), el Consejo de Ministros, siguiendo la práctica habitual, ha acordado la prórroga anual de la participación de las Fuerzas Armadas españolas en todas las misiones que se encuentran desplegadas en el exterior y que han recibido todas ellas la oportuna autorización del Congreso de los Diputados. Además de las misiones de observadores y de mantenimiento de la paz en el marco ONU (Colombia y Líbano), se trata de las siguientes:

- En el marco de las Operaciones de la Unión Europea, continua la participación en la operación 'Atalanta', donde España asumirá el 29 de marzo de 2019 el mando Estratégico-Operacional y por novena vez asumirá el mando de la Fuerza; en la operación 'Sophia', manteniendo el mismo nivel de participación: un buque de la armada y un avión de 
vigilancia o patrulla marítima; en Somalia, donde se prevé un aumento de participación sin sobrepasar los efectivos máximos asignados; en Mali, donde se prevé la asignación del Mando sin que suponga incremento de efectivos; en la República Centroafricana donde se pretende mantener el actual nivel de efectivos; y Bosnia Herzegovina que se mantiene una presencia simbólica. Asimismo, continuarán también los despliegues en Senegal y Gabón de apoyo a las misiones de la UE y de Francia que operan en Mali y República Centroafricana y para este año, se mantiene el compromiso de liderar, como Nación Marco, el Grupo de Combate de la UE (EUBG).

- En relación con los despliegues en el marco de la OTAN, España mantendrá la participación en un Batallón Multinacional liderado por Canadá, con una fuerza formada por un Subgrupo Táctico Mecanizado, unidades de apoyo al combate y unidades de apoyo logístico al combate que está desplegado en Letonia, en el marco de una Presencia Avanzada Reforzada (Enhanced Forward Presence). Se mantendrá el despliegue de una batería 'Patriot' en Turquía; esta aportación se acuerda por seis meses en atención a las necesidades de seguridad. En el Mediterráneo se continuará participando en la operación marítima de seguridad 'Sea Guardian'; así mismo, se seguirán aportando buques de la Armada a las Fuerzas Navales Permanentes Aliadas. La contribución española a esta Operación no sufrirá modificaciones. En Afganistán se mantendrá la participación en la misión 'Resolute Suport' en funciones de asesoramiento y asistencia a los órganos nacionales afganos. Respecto a la lucha contra Daesh, se contribuye con el adiestramiento a la policía iraquí y asesoramiento a autoridades de defensa.

- Asimismo, la Unidad Militar de Emergencia (UME), los Grupos 43 y 45 de Fuerzas Aéreas y otros medios de proyección necesarios, podrán intervenir en operaciones en el exterior para contribuir a la seguridad de los ciudadanos en los supuestos de grave riesgo, catástrofe u otras necesidades, bien en el marco bilateral o formando parte del Mecanismo de Protección Civil de la Unión Europea. 